\title{
PLANIFICACIÓN DE INFRAESTRUCTURAS Y RENOVACIÓN URBANA EN ESPACIOS FERROVIARIOS DESAFECTADOS Revisión de antecedentes europeos desde premisas del Área Metropolitana de Santa Fe (Argentina) \\ Infrastructure planning and urban renovation in vacant railway spaces. European case background review from premises of Santa Fe Metropolitan Area (Argentina)

\author{
Saus, María Alejandra \\ (IHUCSO, UNL - CONICET) \\ produccioncientificaydocencia@gmail.com
}

\begin{abstract}
RESUMEN
Los recientes planes de infraestructuras de transporte (PIT) en América Latina están inscriptos en la dinámica extractiva que domina sus formas de acumulación. Los cambios en las redes ferroviarias se involucran en transformaciones territoriales y urbanas derivadas de la reinversión de parte de la renta agrícola en el campo inmobiliario. Ese problema orienta nuestra indagación sobre sus efectos en el Área Metropolitana de Santa Fe (AMSF), focalizando en los terrenos ferroviarios desafectados por aquellos PIT y en el desarrollo de la industria de la construcción. La ponencia explora las contribuciones y límites de los criterios urbanístico-ferroviarios utilizados en ciudades europeas para ofrecer alternativas al proceso examinado. El trabajo está basado en la técnica del análisis documental y organizado en dos partes: la primera enuncia el problema general y la segunda revisa la casuística. Finalmente, concluye con algunas sugerencias para el AMSF.
\end{abstract}

Palabras clave: planificación de infraestructuras, renovación urbana, terrenos ferroviarios, Santa Fe Bloque temático: espacio público y proyecto urbano en la metrópolis contemporánea.

\begin{abstract}
Recent transport infrastructure plans (TIP) in Latin America are part of the extractive dynamics that dominate their forms of accumulation. The changes in the railway networks are involved in territorial and urban transformations, derived from the reinvestment of part of the agricultural rent in the field of real estate. This problem guides our inquiry into its effects in the Santa Fe Metropolitan Area (SFME), focusing in the railway lands disaffected for those TIP and the development of the construction industry. The paper explores the contributions and limits of urban-railway criteria used in European cities to offer alternatives to the process examined. The work is based on documentary analysis and organized in two parts: the first enunciates the general problem and the second reviews the casuistry. Finally, the paper concludes with some suggestions for SFME.
\end{abstract}

Keywords: infrastructure planning, urban renovation, railway lands, Santa Fe City

Topic: public space and urban project in the contemporary metropolis 


\section{Introducción}

Esta ponencia aborda procesos económicos, políticos y territoriales que están emergiendo en Suramérica con el objetivo de prever su impacto físico en el Área Metropolitana de Santa Fe, Argentina (AMSF). ${ }^{1}$ Las acciones comprenden planes de infraestructuras de transportes que, motivados por dinámicas macroeconómicas y producidos por organismos supranacionales, repercuten a escala local de un modo que trasciende la indagación municipal. Nuestra interpretación de esas dinámicas sugiere la existencia de flujos de materias primas y de capitales que son extraídos e invertidos en la región, generando así una renta agrícola que en gran parte es invertida en las ciudades. Ello sucede porque la industria de la construcción está sustituyendo a la manufactura y el capital financiero ha sido puesto al servicio de financiar sus costos (Coraggio y Muñoz, 2018).

Nuevas redes de ferrocarriles mercantes dinamizan el tráfico exportador de recursos naturales, generando una producción mayormente sojera destinada a los mercados asiáticos, mientras el flujo reingresa a la Argentina a través de acuerdos con China que provee tecnología ferroviaria. Asimismo, los planes dejan infraestructuras obsoletas en áreas centrales urbanas, las que atraen a desarrolladores inmobiliarios nutridos por la renta agrícola. Esto es así porque, si bien ahora asistimos a una fase de recesión, el crecimiento de la actividad económica desde el año 2003 había sido generado por exportaciones debidas al ventajoso precio internacional de los commodities que, sumado a la desconfianza en el sistema financiero tras la crisis del 2001, impulsó la inversión de esos beneficios en la construcción de inmuebles bajo la figura del fideicomiso (Barenboim, 2014). ${ }^{2}$

Las unidades edificadas son un activo financiero para los inversores, pero provocan un incremento en el precio de la vivienda con efectos negativos para las clases medias expulsadas a vivir en el espacio periurbano. En el área central, la tendencia crea un escenario proclive al diseño de proyectos de renovación urbana que tienden hacia la densificación, la verticalización y la segregación socio-espacial. Numerosas operaciones inmobiliarias se asientan en los "vacíos urbanos" heredados (Etulain, 2008), sobre áreas ferroviarias obsoletas que guardan una conexión con el pasado sin establecer otra en el presente debido a las secuelas de la desindustrialización.

En la ciudad de Santa Fe, capital de provincia y cabecera del Ente Coordinador del Área Metropolitana (ECAM), el plan ferroviario dejaría vacantes los cuadros de estaciones y corredores que hoy utiliza el Ferrocarril Belgrano Cargas incluido en los planes supranacionales y nacionales de transporte. Pero cuando esos predios quedan a merced de los operadores inmobiliarios y son apetecidos para crear oportunidades de negocio, el sector privado podría consumir valiosas dotaciones de suelo fiscal. Además, la extensión horizontal en búsqueda de terrenos baratos para la vivienda de clase media y bajo las formas de la ciudad difusa (Ciccolella y Vecslir, 2012), ha creado problemas de movilidad al AMSF debido al uso masivo del automóvil. En tal sentido, el espacio ferroviario desafectado ofrece una llave para mejorar ambas dinámicas: verticalidad y horizontalidad.

Si bien el trabajo está en fase preliminar, abordar los fenómenos suramericanos en su densidad socio-espacial ayuda a entender las dinámicas metropolitanas contemporáneas, su vínculo con las estrategias geopolíticas que están incitando nuevas medidas regionales y nacionales de transporte y su alianza con el sector privado. Ponderamos, entonces, las dinámicas globales de acumulación capitalista y el re-escalamiento estatal que éstas producen (Brenner 2003), reconociendo en la escala local la singularidad de unos procesos ligados a un orden global. Algunos ejemplos europeos son fuentes de proyectos y prácticas que analizamos por el valor asignado al "gesto comparativo" (Robinson, 2016), es decir, trascender el estudio de caso aislado para hallar diálogos teóricos que engloben procesos de acumulación. Desde una metodología cualitativa planteamos dos coordenadas: la variable independiente representa el contexto económico, político y social de Suramérica, mientras la variable dependiente refleja la modalidad urbanístico-ferroviaria de cada caso (Schneider, 2007).

Si bien la recuperación ferroviaria europea respondió a motivaciones diferentes, las ciudades estudiadas integran actuaciones destacadas por la literatura especializada. ${ }^{3}$ Son exponentes de tipologías urbanístico-

\footnotetext{
${ }^{1}$ La ciudad ha sido declarada cabecera del Área Metropolitana (Ley Provincial № 13.532/2016), pero detenta una configuración de tamaño medio y escasa continuidad física en su conglomerado (alrededor de 600.000 habitantes).

${ }^{2}$ Si bien algunos economistas afirman que la correlación entre renta agropecuaria e industria de la construcción aún no ha sido demostrada numerosos autores la consideran un hecho consumado (Elinbaum y Barenboim, 2018; Rausch et.al., 2019).

${ }^{3}$ La autora fue Investigadora/Colaboradora de la Biblioteca/Archivo Histórico Ferroviario de la Fundación de los Ferrocarriles Españoles.
} 
ferroviarias que hemos dividido en cuatro categorías. Para las estaciones de viajeros: 1) creación de suelo en desarrollos urbanos a escala regional, 2) renovación de áreas centrales a partir de la construcción de nuevos edificios, 3) integración de usos terciarios a las estaciones históricas. En los corredores ferroviarios: 4) construcción de viaductos, desvíos, soterramientos y/o integración en superficie. Entre los ejemplos elegidos para la ponencia se hallan ciudades intermedias (Bellet y Llop, 2004) que cumplen esas condiciones: Alto Taastrup (Dinamarca), Lille (Francia), Zúrich (Suiza) y Córdoba (España). ${ }^{4}$ El trabajo emplea la técnica del análisis documental y se organiza en dos partes: la primera introduce el contexto suramericano, argentino y del AMSF, y la segunda revisa las actuaciones europeas concluyendo con algunas sugerencias generales.

\section{La integración regional y su impacto local}

En el año 2000 se iniciaron estudios para la renovación de infraestructuras tendientes a la integración regional y a la reducción de los costos logísticos del transporte de cargas. Entonces fueron creados la Iniciativa para la Integración de la Infraestructura Regional Suramericana (IIRSA) y en 2009 el Consejo Suramericano de Infraestructura y Planeamiento (COSIPLAN). ${ }^{5}$ Diversos autores han criticado esos programas ya que los organismos supranacionales no estarían previendo un desarrollo territorial equitativo (Rascovan, 2016). Así, los proyectos idean infraestructuras para fortalecer las vías de circulación entre los grandes polos productivos y los principales puertos de exportación, marginando a las economías más pequeñas.

Hacia 2010 se firmó en la Argentina un convenio entre la Secretaría de Transporte de la Nación y la empresa China Machinery and Engineering Corporation (CMEC) para renovar 1600 km del Ferrocarril Belgrano Cargas. Esa iniciativa mejoraría la vinculación entre las provincias de Salta, Chaco y Santa Fe, elevando las toneladas transportadas hacia los puertos de la Región Metropolitana de Rosario (Fig. 1). Esa recuperación ferroviaria integra un plan contemplado en los ejes del IIRSA, pero las acciones también tienen impacto urbanístico puesto que se procura evitar demoras y accidentes en los pasos a nivel. El plan Circunvalación Ferroviaria de la Ciudad de Santa Fe fue planeado en 2016 por iniciativa de Trenes Argentinos Infraestructura del ahora Ministerio de Transporte de la Nación. ${ }^{6}$ La traza se extiende entre la ciudad de Santo Tomé y la localidad de Laguna Paiva y evade la trama urbana dejando a su interior un paquete de terrenos vacantes. ${ }^{7}$

\footnotetext{
${ }^{4}$ En el sistema urbano argentino son aglomeraciones de tamaño intermedio las ciudades de entre 50.000 y 999.999 habitantes (Vapñasky y Gorojovsky,1990; Erbiti, 2007). Esa clasificación varía según el autor y cambia para la ciudad media europea que puede tener entre 20.000 y 500.000 (Feliu, 2007). Al ser un estudio comparado hemos decido solapar ambos criterios. El enfoque está flexibilizado por un abordaje cualitativo de las funciones de intermediación (Bellet y Llop, 2004). Por ahora, los casos son: Valencia, Sevilla, Zúrich, Córdoba, Valladolid, Lille, Oviedo, Ginebra, Burgos, Santander, Gerona, Palencia, Segovia, Alto Taastrup.

${ }^{5}$ Ese Consejo está integrado por los ministerios de transporte y obras públicas de los países integrantes de la UNASUR (www.iirsa.org).

${ }^{6}$ El Proyecto de Recuperación y Mejoramiento del Ferrocarril General Belgrano comenzó cuando Transporte era una Secretaría del Ministerio de Planificación Federal, Inversión Pública y Servicios que también impulsó el Plan Estratégico Territorial en 2004.

${ }^{7}$ Licitación Pública LP 16-ADIF- 2016. Fuente: https://servicios.adifse.com.ar/site/licitaciones_nuevas/PCP\%20LP\%2016-2016.pdf
} 


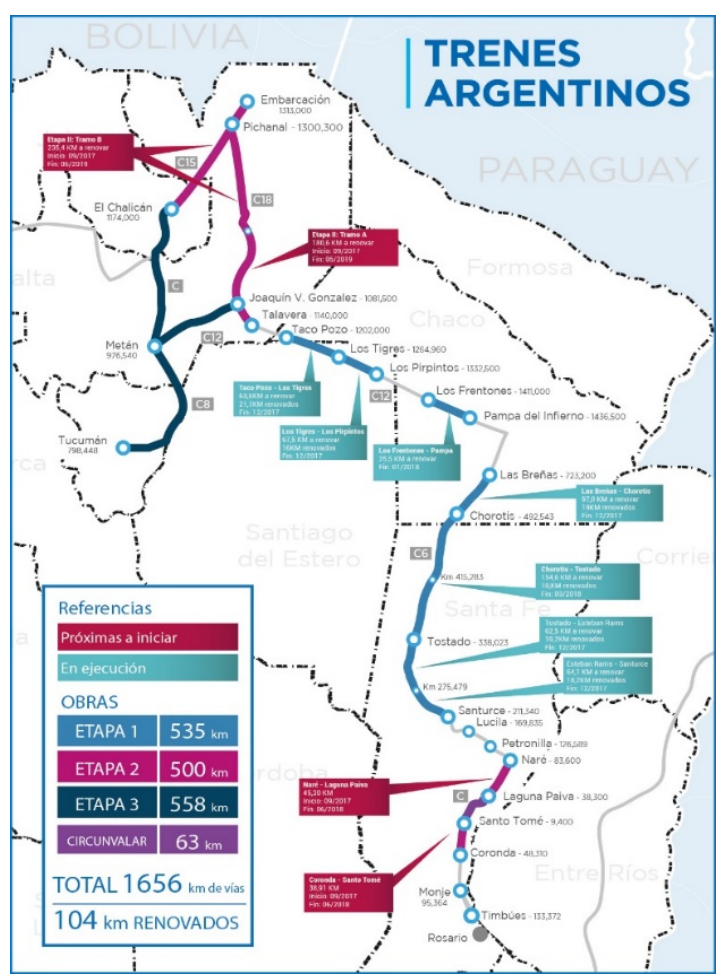

Fig. 1. Recuperación del Ferrocarril Belgrano Cargas (Consulta: 22/7/2019). Fuente: https://www.airedesantafe.com.ar/noticiasregionales-santa-fe/trenes-formaciones-ciudad/

La variante ferroviaria (Fig. 2, rojo) tendrá efectos territoriales y producirá externalidades a escala local. Por ello, más allá de los objetivos directos -que son los derivados del programa ferroviario- ¿cuáles serían los efectos externos, es decir, las utilidades o desutilidades que podría ocasionar a la ciudad? Mejorar una infraestructura de transporte tiene objetivos capaces de beneficiar a usuarios, transportistas y administraciones, pero también potenciales externalidades sobre los residentes linderos a las vías, las explotaciones agrícolas y las actividades comerciales que experimentan oportunidades o dificultades por acciones impuestas desde las escalas global, nacional o regional sin demasiada anuencia local (Serrano, 1988).

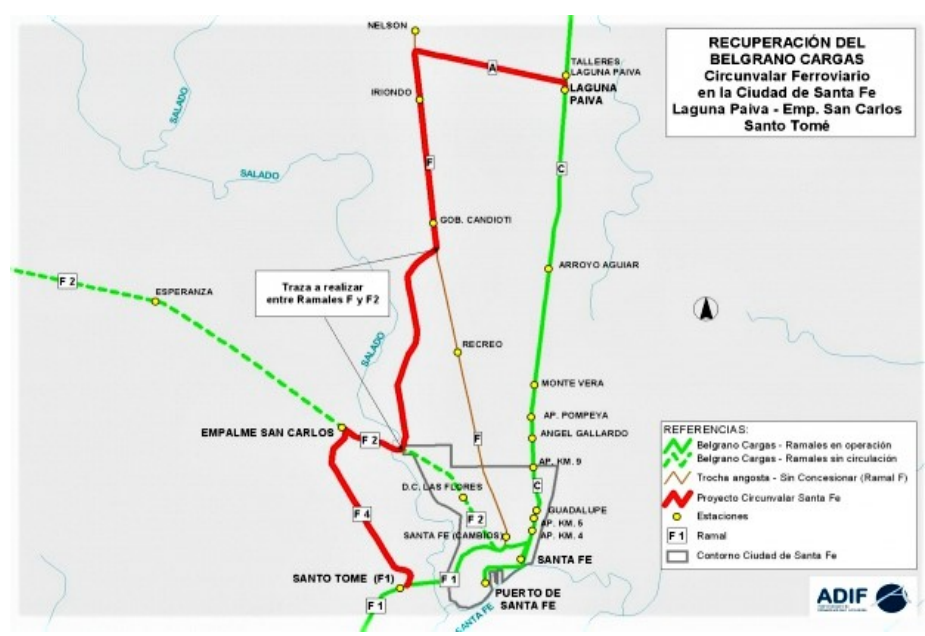

Fig. 2. Circunvalación Ferroviaria (Consulta: 10/2/2019). Fuente: https://www.argentina.gob.ar/trenes-argentinos-infraestructura 
En Santa Fe se ha planeado una urbanización sobre el cuadro de estación desafectado (Fig. 3), a través de un acuerdo entre la Agencia de Administración de Bienes del Estado (AABE) y la Municipalidad. ${ }^{8}$ Son 24 ha céntricas destinadas a impulsar desarrollos inmobiliarios, un distrito tecnológico y la apertura de calles para restablecer la conectividad este-oeste. Si bien fue acordada la transferencia del edificio de viajeros y algunos terrenos del entorno, su rol de centro de exposiciones muestra que ha sido descartado el uso ferroviario. Paralelamente, la apertura de calles entra en colisión con el proyecto de conservar una vía norte-sur para el servicio de cercanías. Mantener la vía también problematiza construir en alta densidad, ya que eso exige aumentar las distancias de las edificaciones linderas por cuestiones acústicas (Dómine, 1994). Todas esas ideas aisladas demuestran que, hasta ahora, no ha sido pensado integralmente el problema urbano-ferroviario.

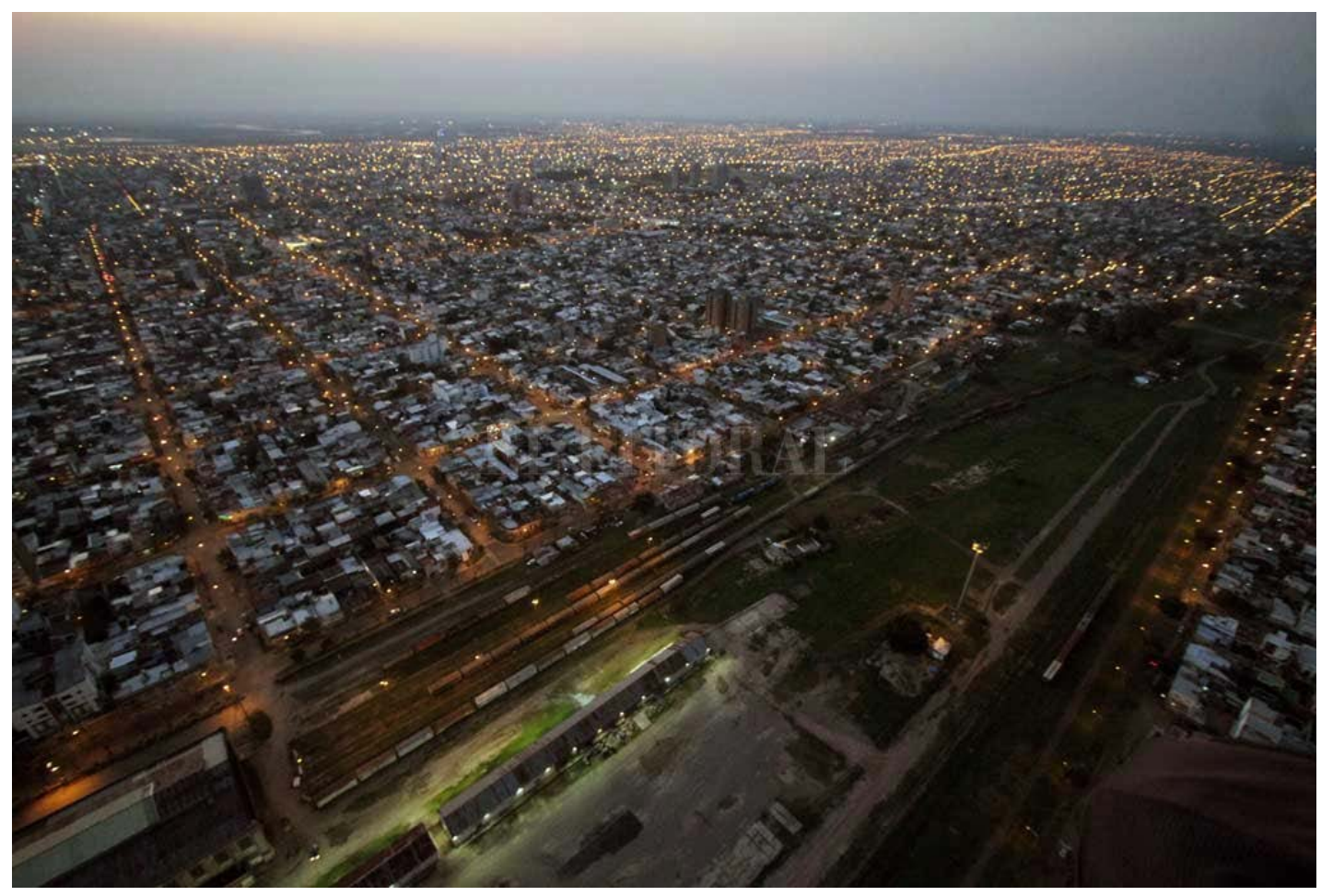

Fig. 3. Cuadro de la estación Santa Fe del Ferrocarril Belgrano. (Consulta: 10/2/2019) Fuente:

https://www.ellitoral.com/index.php/id um/185574-boom-de-desarrollos-inmobiliarios-en-espacios-ferroviarios-en-desuso-la-nacion-sedesprende-de-tierras-en-la-ciudad-area-metropolitana.html?utm source=relacionadas

La municipalidad deberá ahora ampliar el Reglamento de Ordenamiento Urbano (ROU) porque el sector este del predio corresponde a "terrenos fiscales", de modo que la normativa sería modificada ad hoc con la coerción inmobiliaria instalada y el Estado nacional intentando rentabilizar la operación. En simultáneo, se ha presentado el Programa de Resguardo y Reutilización de Vías Ferroviarias, una iniciativa del ECAM puesta a consideración de las autoridades nacionales de la AABE. El plan fomenta políticas de escala metropolitana para resguardar y reutilizar el inventario ferroviario disponible, dejando clara su potencialidad para el transporte de cercanías. No obstante, ha sido divorciado el cuadro de la estación -integrable al mercado inmobiliario- de los corredores que serían preservados para la circulación de trenes, desarticulando así la complejidad original del sistema. ${ }^{9}$

${ }^{8}$ Fuente:https://www.ellitoral.com/index.php/id_um/185138-se-firmo-el-acuerdo-para-urbanizar-los-terrenos-de-la-estacion-belgranoentre-nacion-y-el-municipio-area-metropolitana.html

${ }^{9}$ Fuente:https://www.ellitoral.com/index.php/id_um/186499-presentaron-un-programa-para-reutilizar-la-traza-ferroviaria-oportunidad-dedesarrollo-para-el-gran-santa-fe-area-metropolitana.html 


\section{Algunas experiencias urbanísticas internacionales}

Revisaremos cuatro operaciones urbanístico-ferroviarias para analizar sus criterios de actuación, limitaciones y potencialidades. Son actuaciones que van desde los convenios bilaterales entre la compañía ferroviaria y el municipio hasta los multilaterales representados por consorcios, ya que la complejidad de esa gestión excede las capacidades locales y desafía los modelos tradicionales (Santos, 2007). El primer aprendizaje, entonces, es la necesidad de coordinar a todos los organismos públicos, tanto en relación al escalamiento estatal como para la acción público-privada. Puesto que la arquitectura institucional es penetrada por el capital global y los niveles gubernamentales son permeables, es preciso que los problemas de gobernanza metropolitana sean abordados en su multiescalaridad, no como meros asuntos de regulación municipal (Brenner, 2003).

Los terrenos ferroviarios suelen generar oportunidades para la satisfacción de requerimientos urbanos. Una premisa fundamental es preservarlos, pero eso crea conflictos entre la administración nacional que pretende rentabilizarlos y los municipios que buscan corregir déficits urbanísticos e introducir mejoras en espacios verdes, movilidad, vivienda y pasos a desnivel. Por eso, reflexionamos sobre el ferrocarril y la mejor utilización de los terrenos vacantes, ya que "La adopción de usos del suelo congestivos y generadores de efectos externos no debe ser la pauta prioritaria para la reutilización de espacios ferroviarios obsoletos, ni aún con la excusa de que este es el medio de financiar mejoras en la red o en el servicio..." (Serrano, 1994: 72).

Del estudio de casos europeos hemos extraído algunas pautas de ordenamiento, gestión y financiamiento en ciudades intermedias que han desarrollado estrategias para incorporar nuevos trenes a finales del siglo XX. En términos estructurales, esas ciudades tienen problemas de transporte parecidos a las grandes, pero poseen mayores dificultades de gobernanza y desventajas para influir en los niveles superiores de gobierno. Además, Feliu (2007) sostiene que la dimensión de la ciudad tiene estrecha relación con los problemas financieros. Empero, muchas de ellas han aprovechado la incorporación de nuevas tecnologías ferroviarias para impulsar desarrollos urbanos debido al declive de la actividad industrial, la disponibilidad de capital financiero global, la demanda de un servicio eficiente de viajeros de cercanías, las directrices territoriales y ambientales de la Unión Europea (UE). Pero lo más determinante en esas acciones ha sido la introducción del Tren de Alta Velocidad (TAV) de la Red Trans-europea de Transporte de la que Francia sería pionera en 1981.

Las redes del TAV fueron ideadas para el tráfico de pasajeros entre grandes áreas metropolitanas, por lo cual, las ciudades intermedias quedaron al margen de sus servicios. Actualmente, la situación está siendo revertida, pero esas aglomeraciones nunca están exentas de conflictos. Otra debilidad radica en la filosofía de las decisiones tomadas en las disputas entre municipio y administración ferroviaria. Si bien los gobiernos locales suelen preferir que las estaciones de pasajeros sean ubicadas en áreas centrales para impulsar la renovación, la gestión del ferrocarril tiende a priorizar las construcciones periféricas en localidades menores a 100.000 habitantes (Ribalaygua, 2005). Entonces, a diferencia de Suramérica, las motivaciones de la recuperación ferroviaria europea dependieron de los trenes de viajeros, no de cargas, pero en ambos casos existe un impulso que despliega consecuencias urbanísticas y proyectos que la experiencia europea puede iluminar.

Los casos escogidos son pioneros, exitosos y representativos de las cuatro categorías ya mencionadas. Alto Taastrup encarna los efectos de la planificación metropolitana a partir de una nueva estación y urbanización. Lille logró ganar una estación central y crear tejido urbano. La ciudad de Zúrich pudo modernizar su antigua estación junto a la regeneración del entorno. Córdoba eliminó la barrera creada por las vías y construyó un paseo público. Analizar esas operaciones aporta referencias para los desafíos de gobernanza del AMSF. No obstante, advertimos que desde la perspectiva ferroviaria la estación es un nodo y para el enfoque de la ciudad se constituye en un lugar y debemos transitar hacia la integración de ambos criterios (Bertolini y Spit, 2008).

1) El desarrollo de la región metropolitana de Copenhague responde a un plan de la década de 1940 llamado Fingerplanen. Pretendía controlar el crecimiento con urbanizaciones contiguas a las estaciones suburbanas y zonas verdes entre los dedos de la mano que simula la planta. Las estaciones fueron proyectadas por los Ferrocarriles del Estado de Dinamarca (DSB) para descongestionar el tráfico de la estación central y el tren opera como articulador para un desarrollo regional sin barreras físicas (Bjarrum, 1994). Alto Taastrup es uno 
de los tres puntos de confluencia urbanístico-ferroviaria planeados en la década del setenta sobre la principal línea férrea nacional de largo recorrido. Un concurso de proyectos determinó las actuaciones específicas sobre un plan director de $200.000 \mathrm{~m}^{2}$, de los cuales $60.000 \mathrm{~m}^{2}$ fueron destinados a vivienda, $36.000 \mathrm{~m}^{2}$ a comercios y el resto a oficinas. Con el edificio emblemático de la estación como elemento estructurante de la urbanización (1986), esta operación dio inicio a una fructífera cooperación entre la DSB y el ayuntamiento (Fig.4).

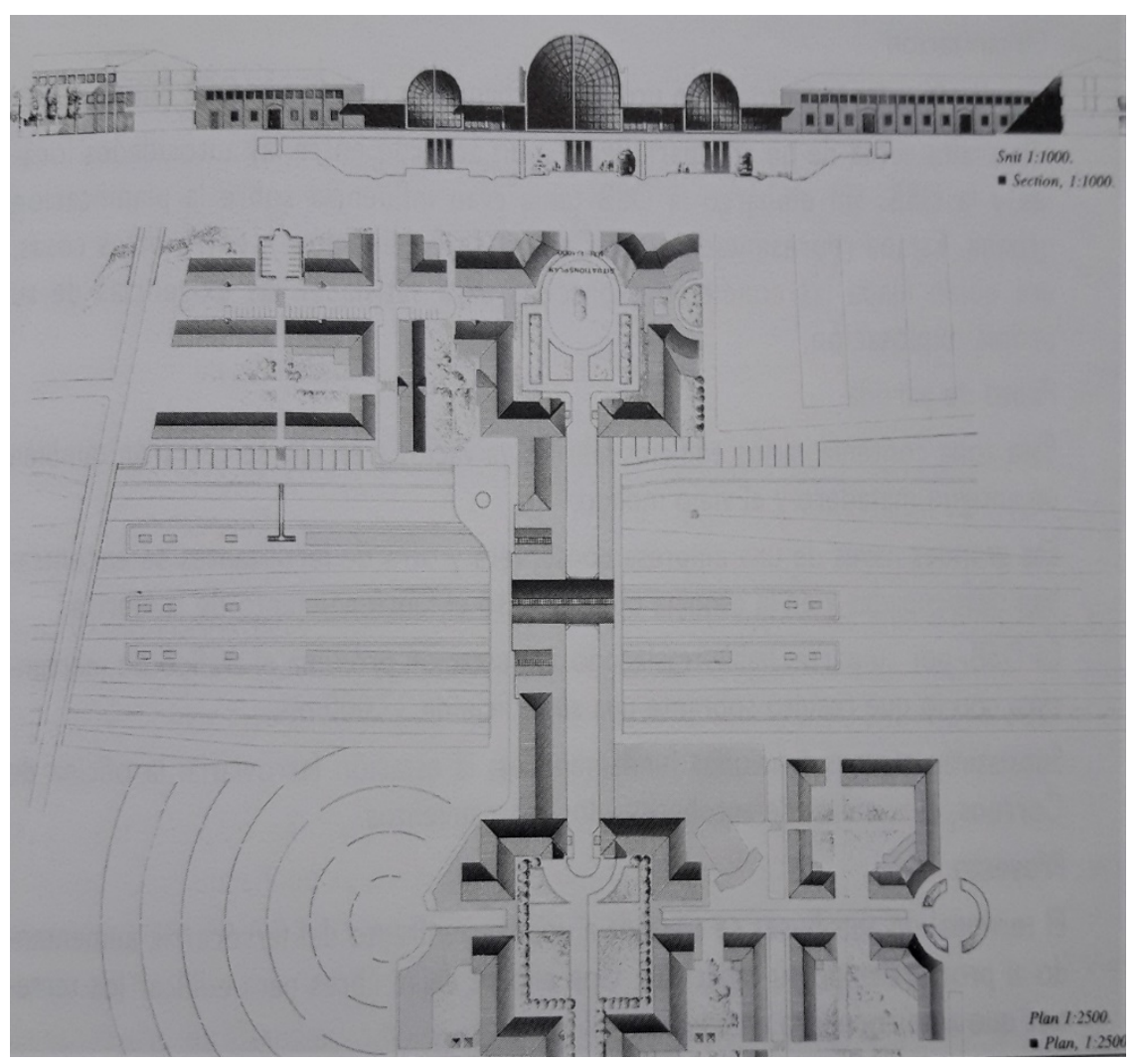

Fig. 4. Proyecto de estación y urbanización Alto Taastrup. Fuente: BJARRUM, K. (1994), p.103

2) A diferencia de la planificación estatal del caso danés, la entrada del Train à Grande Vitesse (TGV) en Francia tuvo un sesgo liberal compatible con la adaptación de la red de estaciones centrales decimonónicas por la Société Nationale des Chemins de Fer Français (SNCF). En general, fueron malogradas las actuaciones en ciudades más pequeñas que no alcanzaron el desarrollo previsto (Facchinetti, 2005). Lille es una excepción por ser una estación nueva construida en el centro de una ciudad intermedia. El proyecto fue una estrategia local para acercar a la ciudad el nudo señalado por razones económicas para la conexión internacional. A partir de la disponibilidad de 70 ha vacantes y otras 50 liberables junto a la antigua estación, se optó por impulsar un desarrollo urbanístico con arquitectura de Rem Koolhaas (Harrus, 1994). La operación contempló torres de oficinas y un hotel sobre la estación, un palacio de congresos, centro comercial, escuela y viviendas en una superficie de $300.000 \mathrm{~m}^{2}$. Fue gestionada por un consorcio llamado Sociedad Anónima de Economía Mixta, con un $34 \%$ de inversiones públicas y el $66 \%$ restante proveniente de la participación privada (Fig.5). 


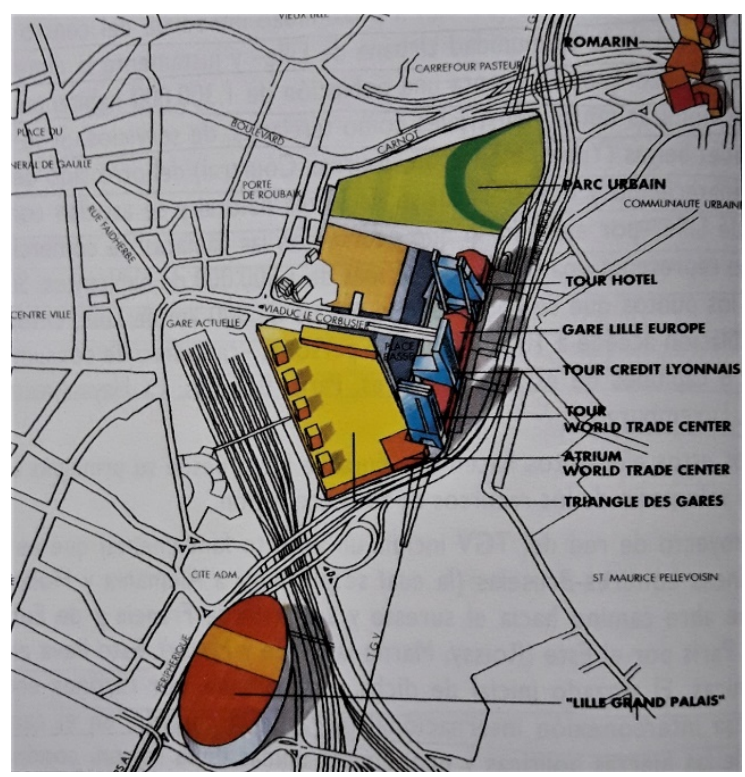

Fig. 5. Proyecto de estación y urbanización en Lille. Fuente: HARRUS, K. (1994), p.220

3) La transformación de la Estación Central de Zúrich es un modelo de equilibrada integración de la actividad comercial al edificio histórico de viajeros, sin menoscabar una buena dotación de espacios públicos y acertadas estrategias de intermodalidad. La incorporación de usos terciarios fue cardinal en el concepto de renovación iniciado en 1967 y culminado en la década del ochenta por la compañía de Ferrocarriles Federales Suizos (SBB). El centro comercial fue situado en el nivel intermedio en un lugar privilegiado de paso de peatones hacia la plataforma de distribución del intercambiador de la estación. La idea fue generar atractivos para disminuir el uso del transporte automotor que había viabilizando la extensión residencial hacia la periferia con la inevitable congestión del tráfico central (Stieger, 1994). Las escaleras mecánicas juegan un rol vital en la organización interna, como así también la conservación del patrimonio ferroviario, cuya conjunción con lo moderno es un rasgo típicamente suizo ya que las estaciones nuevas de Lucerna o Berna son excepcionales (Fig. 6).

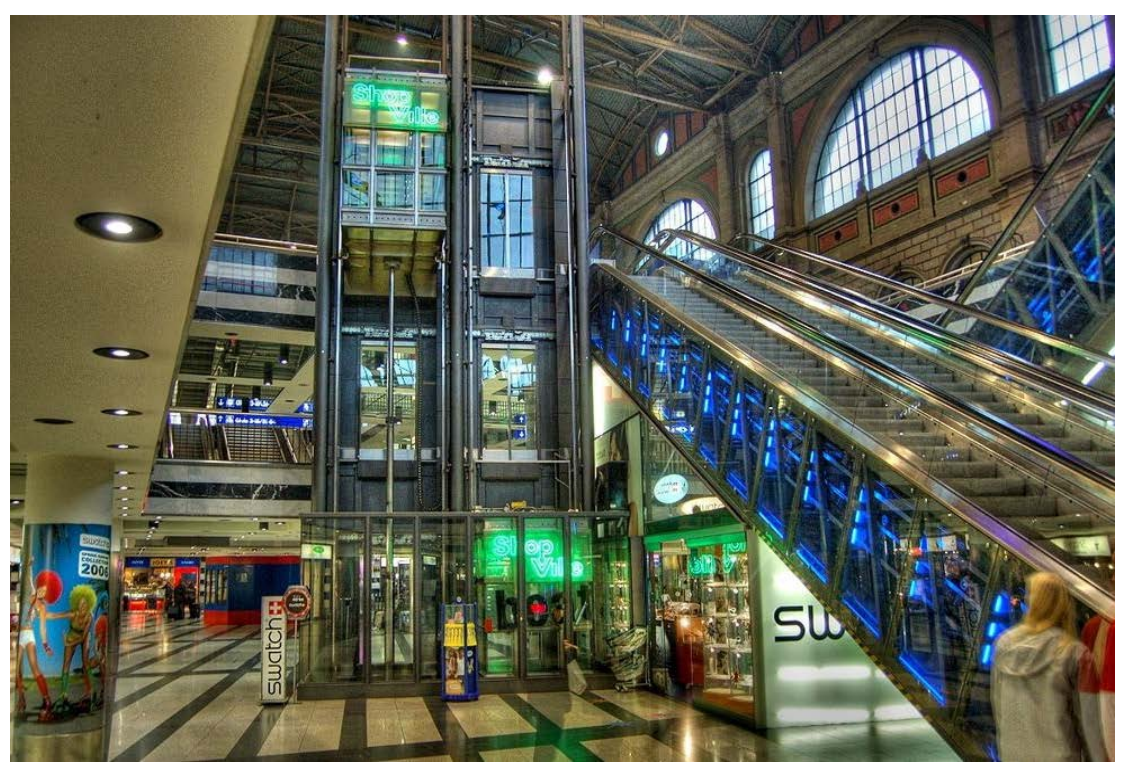

Fig. 6. Centro comercial (Consulta: 28/2/2019). Fuente: https://serturista.com/suiza/compras-y-viajes-en-la-estacion-central-de-zurich/

4) Acerca de las actuaciones sobre los enlaces en esta ponencia hemos decido exponer sólo un ejemplo de soterramiento. Se trata de estrategias para eliminar el efecto barrera, es decir, la falta de continuidad urbana 
debida a la interrupción provocada por las vías (López, 2005). Córdoba fue una ciudad media precursora en el soterramiento de 2,5 km de vías férreas, comenzando en 1989 a partir de un convenio entre el Ministerio de Transportes, la Junta de Andalucía, la Red Nacional de los Ferrocarriles Españoles (RENFE) y el Ayuntamiento (Santos, 2007). Fueron vendidos al municipio los terrenos liberados para financiar luego las obras nuevas. Sobre la infraestructura de alta velocidad fue ejecutado un paseo recreativo, deportivo y cultural, asegurando una gran dotación de espacios públicos en un parque lineal y la supresión de todos los pasos a nivel. El ayuntamiento hizo fuertes desembolsos al prescindir de la opción menos costosa de integrar en superficie, pero la obra fue un éxito en la preservación pública del suelo y la promoción turística (Fig. 7).

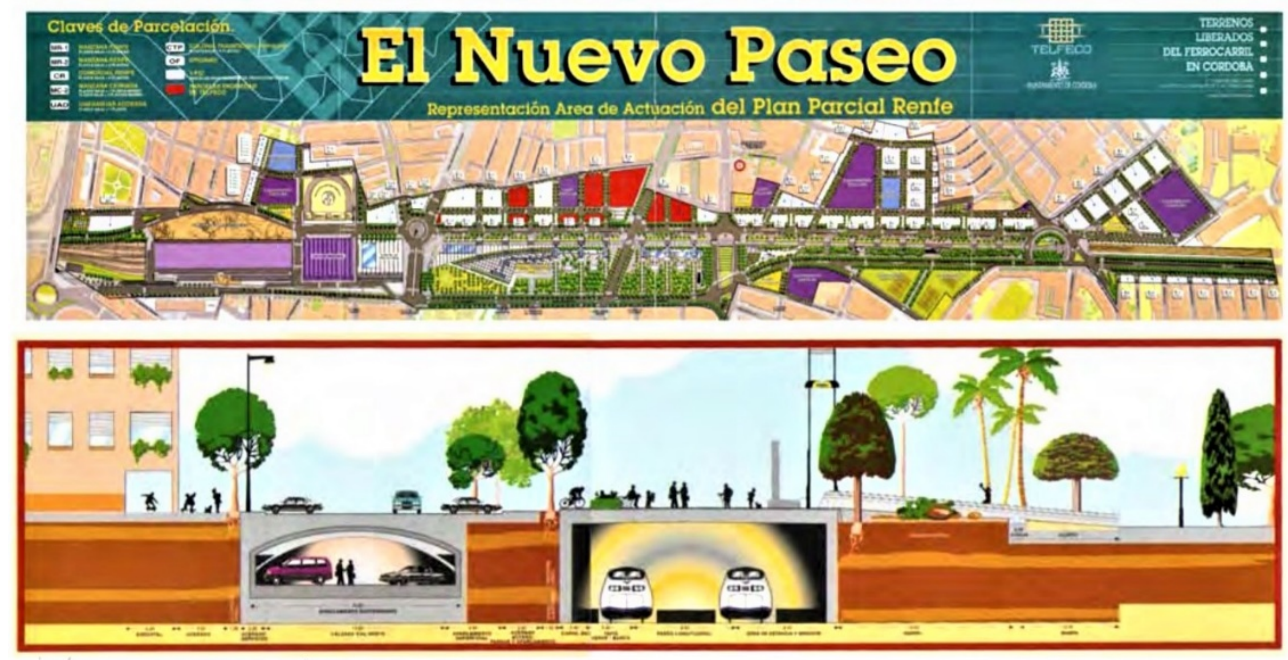

Fig. 7. Proyecto de soterramiento para Córdoba. Fuente: LAGUIA, V. (2015), p.15

El soterramiento ha sido posible también en Madrid, Sevilla y Barcelona (1992) donde el clima creado por eventos internacionales concentró recursos suficientes al incorporar la Alta Velocidad Española (AVE). Sin embargo, debido a esas obras se generalizaron pedidos de soterramiento incluso en pequeños ayuntamientos cuyos mercados inmobiliarios no podían financiarlos. Esas acciones empujan a las sociedades gestoras hacia el capital financiero teniendo como presunta garantía las futuras plusvalías inmobiliarias. Hoy, la crisis económica que atraviesa Europa tiene a las sociedades muy endeudadas en ciudades medias como Valladolid que ahora debe reestructurar su deuda y abandonar la idea del soterramiento (Santos, 2016). ${ }^{10}$

\section{Conclusiones}

Los procesos macroeconómicos que impulsan el mejoramiento de infraestructuras en Suramérica convergen en la exportación de productos primarios. En la Argentina, tal situación interpela nuestro interés disciplinar porque las redes de transporte proyectadas generan mutaciones urbanas con complejidades asociadas a la inversión de renta agrícola en bienes inmuebles. En el AMSF esas dinámicas hacen del espacio ferroviario desafectado un área vulnerable ¿qué destino tendrían las infraestructuras obsoletas ante la avidez especulativa en tensión con el derecho a la vivienda y a la movilidad sostenible? Su posible entrega acrítica a los agentes inmobiliarios y las disputas competenciales han motivado esta ponencia sobre modelos de gestión exógenos.

Entre las diferencias comparadas se hayan los tipos de transporte impulsados en ambos contextos. Mientras el renacimiento de los ferrocarriles en Suramérica responde al tráfico de cargas, la recuperación ferroviaria europea dependió del TAV y del ferrocarril convencional de cercanías. Además, las medidas supranacionales de la UE han empujado a los países menos dinámicos hacia la conectividad trans-europea, prosiguiendo cada

\footnotetext{
${ }^{10}$ El túnel madrileño, concluido antes de la crisis del 2008, fue realizado para una extensión mínima de 4 km. Hoy, ni Buenos Aires está pudiendo terminar el descomunal tunelado de $18 \mathrm{~km}$ del Ferrocarril Sarmiento, debiendo replantear las obras hacia la opción del viaducto. Fuente: https://www.clarin.com/economia/gobierno-analiza-transformar-viaducto-soterramiento-ferrocarril-sarmiento_0_i_9jAgrvX.html
} 
uno con sus redes internas (TGV, AVE). Otras divergencias anidan en la escala de las ciudades intermedias, puesto que las nuestras son más grandes y menos densas, hecho que repercute negativamente en el desarrollo de potenciales ferrocarriles de viajeros. No obstante, las operaciones de integración del ferrocarril en el medio urbano ofrecen referencias de importancia estratégica en torno a la gestión del espacio ferroviario desafectado.

En el AMSF los terrenos vacantes podrían ser claves para activar sectores degradados y dotar a otros carentes de infraestructuras y servicios domiciliarios. Esos espacios estarían en condiciones de recomponer el orden planificación-urbanización-edificación que ha sido invertido por la urgencia de las clases medias de adquirir solares periféricos, debido al aumento de los precios en las unidades de vivienda. Eso ha traído también la especulación inmobiliaria y el problema derivado del incremento de la movilidad que congestiona a la ciudad central y conspira contra su calidad ambiental. Pareciera ser un objetivo atinado de gestión metropolitana desarrollar una estrategia urbanística que aprovechara el patrimonio ferroviario para dinamizar barrios céntricos con estaciones renovadas capaces de proveer, además, nuevos nodos de transporte a una ciudad dispersa.

Reciclar los edificios de viajeros sería una manera de preservarlos para dar vitalidad a espacios subutilizados o degradados. Descartando la opción de la alta velocidad cuya introducción no parece factible ni justificable en la Argentina, las estaciones históricas podrían albergar ferrocarriles convencionales que ayudaran a disminuir el uso del automóvil. Asimismo, provistas de locales comerciales y de una buena coordinación entre modos de transporte, éstas podrían generar formas rentables de recuperación de las inversiones con la venta de locales y la promoción de eventos. En el entorno podrían emplazarse desarrollos urbanos de media y alta densidad, no así junto a las vías ya que las torres requieren de especiales tratamientos acústicos. Además, debería remediarse el continuo urbano quebrado por las vías con un tratamiento de bordes, debido a que modificar la cota del ferrocarril es una erogación muy grande para los recursos de una ciudad intermedia.

Para todo ello sería imprescindible que las acciones fueran concertadas por todas las administraciones públicas con proyectos directores y sectoriales que priorizaran el espacio público y la vivienda protegida. Una sociedad mixta podría tramitar las obras, ya que su complejidad excede las capacidades municipales de financiamiento y gestión. Ello es sustancialmente importante al momento de pensar en una opción para permeabilizar barreras físicas. Son realmente excepcionales los casos donde los costos de un túnel han podido ser afrontados por una ciudad intermedia. Incluso en las grandes ciudades exitosas esa opción se ha adoptado para tramos muy cortos y genera unos trastornos al ferrocarril que sólo son justificables ante una gran obra de transporte.

\section{BIBLIOGRAFÍA}

BARENBOIM, C. A. (2014). Evolución histórica de la costa central y su vinculación con el gran proyecto urbano de Puerto Norte en la ciudad de Rosario, Argentina. Revista Iberoamericana de Estudios Municipales (Santiago de Chile), 10, 61-81.

BELLET SANFELIU, C. y LLOP TORNÉ, J. M. (2004). Miradas hacia otros espacios urbanos: las ciudades intermedias. Scripta Nova (Barcelona), VIII (165), 1-28.

BERTOLINI, L. y SPIT, T. (2005). Cities on Rails. The redevelopment of railway stations areas. London and New York: E and FN SPON.

BJARRUM, K. (1994). Ejemplos daneses. En AA.VV. Integración del ferrocarril en el medio urbano. Madrid: Fundación de los Ferrocarriles Españoles (FFE).

BRENNER, N. (2003). La formación de la ciudad global y el re-escalamiento del espacio del Estado en la Europa Occidental post-fordista. Eure (Santiago de Chile), 29 (86), 5-35.

CICCOLELLA, P. y VECSLIR, L. (2012). Dinámicas, morfologías y singularidades en la reestructuración metropolitana de Buenos Aires. RIURB (Barcelona), 8, 23-41. 
CORAGGIO, J. L. y MUÑOZ, R. (dirs.) (2018). Economía de las ciudades de América Latina hoy I. Los Polvorines: Universidad Nacional de General Sarmiento.

DÓMINE REDONDO, V. (1994). Soluciones integradas y eficiencia presupuestaria. En AA.VV. Integración del ferrocarril en el medio urbano. Madrid: FFE.

ELINBAUM, P. y BARENBOIM, C. (2018). Efectos metropolitanos de una política de vivienda mixta. El caso del ProCreAr. Cuadernos de Vivienda y Urbanismo (Colombia), 11 (21),1-20.

ERBITI, C. (2007). Transformaciones del sistema urbano argentino a fines del siglo XX: desafíos para la gestión del territorio. IV Seminario Ordenamiento Territorial, Ordenamiento Territorial y Problemáticas Urbanas.

ETULAIN, J. C. (2008). La gestión urbanística mixta de intervención en vacíos urbanos de ciudades centrales. Caracterización, análisis e instrumentos. Estudios del Hábitat (La Plata), 10: 45-55.

FACCHINETTI-MANNONE, V. (2005). Efectos espaciales de las estaciones del TGV implantadas en la periferia de las ciudades pequeñas. IT (Borgoña), 70, 22-27.

FELIU TORRENT, J. (2007). El desarrollo local en la ciudad media europea ante los proyectos de T.A.V. Estudios Geográficos (Madrid), LXVIII (262), 65-90.

HARRUS, M. (1994). Un caso especial de desarrollo de suelo y actividad: Lille. En AA.VV. Integración del ferrocarril en el medio urbano. Madrid: FFE.

JIMENEZ AGUILAR, J. M. (1994). Actuaciones urbanístico-ferroviarias: referencias para una integración. En AA.VV. Integración del ferrocarril en el medio urbano. Madrid: FFE.

LAGUIA, V. (2015). L'eau Anthropique. École Doctorale de Geógraphie de Paris. Espaces, Sociétés Aménagent, Université Paris 1 - Panthéon Sorbonne.

LAN, C. (2017). Diplomacia china del ferrocarril en América Latina. Revista Orientando (Veracruz), 7 (14), 120147.

LÓPEZ LARA, E. (2005). Urbanismo y Ferrocarril. PH Boletín del Instituto Andaluz del Patrimonio Histórico (Sevilla), 13 (55), 15-22.

PRADILLA COBOS, E. (2018). Formas productivas, fracciones de capital y reconstrucción urbana en América Latina. En CORAGGIO, J. L. y MUÑOZ, R. (dirs.) (2018). Economía de las ciudades de América Latina hoy I. Los Polvorines: Universidad Nacional de General Sarmiento.

RASCOVAN, A. (2016). La infraestructura y la integración regional en América del Sur. Una visión geopolítica de los proyectos ferroviarios en el marco de IIRSA-COSIPLAN. Relaciones Internacionales (La Plata), 25 (51), 59-80.

RAUSCH, G. et. al. (2019). Concentración/dispersión en Santa Fe, Argentina: problemáticas e interrogantes sobre los procesos urbanos contemporáneos en una ciudad media de América Latina. Cuadernos de Geografía: Revista Colombiana de Geografía (Bogotá), 28 (1), 66-88.

RIBALAYGUA BATALLA, C. (2005). Alta velocidad ferroviaria y ciudad: estrategias de incorporación de las nuevas estaciones periféricas francesas y españolas. Red de Cuadernos de Investigación Urbanística 44. Madrid: Instituto Juan Herrera.

ROBINSON, J. (2016). Ciudades en un mundo de ciudades: el gesto comparativo. Andamios (México), 13 (32), 163-210.

SANTOS Y GANGES, L. (2007). Urbanismo y ferrocarril. La construcción del espacio ferroviario en las ciudades medias españolas. Madrid: FFE.

SANTOS Y GANGES, L. (2016). Autofinanciación de los grandes proyectos urbanos: las sociedades de integración ferroviaria, de la entelequia embarazosa al riesgo fatal. Revista Urban (Madrid), 10 (11), 40-65. 
SCHNEIDER, C. (2007). Participación ciudadana en los gobiernos locales: contexto político y cultura política. Un análisis comparativo de Buenos Aires y Barcelona. Tesis de Doctorado, Universitat Pompeu Fabra, Barcelona.

SERRANO RODRÍGUEZ, A. (1988). Efectos territoriales y urbanísticos de los ferrocarriles: aportaciones metodológicas. En AA.VV. Ferrocarril, Urbanismo y Territorio. Madrid: FFE.

SERRANO RODRÍGUEZ, A. (1994). El futuro: ferrocarriles integrados en el medio urbano. En AA.VV. Integración del ferrocarril en el medio urbano. Madrid: FFE.

STIEGER, H. (1994). Zurich, estación central. Espacio comercial privilegiado. En AA.VV. Integración del ferrocarril en el medio urbano. Madrid: FFE.

VAPÑARSKY, C. y GOROJOVSKY, N. (1990). El crecimiento urbano en la Argentina. $1^{\text {a }}$ ed. Buenos Aires: Grupo Editor Latinoamericano (GEL).

VEGA PINTADO, P. (1994). Balance social de las actuaciones urbanístico ferroviarias. En AA.VV. Integración del ferrocarril en el medio urbano. Madrid: FFE. 